\title{
On strongly starlike and strongly convex functions with bounded radius and bounded boundary rotation
}

\author{
Sabir Hussain ${ }^{1 *}$ and Khalil Ahmad ${ }^{2}$
}

"Correspondence:

sabiriub@yahoo.com;

sh.hussain@qu.edu.sa

'Department of Mathematics, College of Science, Qassim

University, Buraydah, Saudi Arabia

Full list of author information is

available at the end of the article

\begin{abstract}
In this paper, we introduce and investigate new classes of normalized analytic functions in an open unit disk with bounded radius and bounded boundary rotation by using the subordination. We discuss inclusion results, co-efficient bounds, growth and distortion theorems of the classes. Moreover, we compute the radii of strong starlikeness, convexity and starlikeness of the classes. It is interesting to mention that most of our findings are best possible as compared to the existing results in the literature.
\end{abstract}

MSC: $30 C 45 ; 30 C 50$

Keywords: Subordination; Strongly convex function; Janowski function; Bounded boundary rotation

\section{Introduction and preliminaries}

We represent $\mathcal{A}$ as the class of functions $f(z)$, which is analytic in $\mathrm{E}=\{z \in C:|z|<1\}$. Then, for each $z \in E, f(z)$ has the form

$$
f(z)=z+\sum_{n=2}^{\infty} a_{n} z^{n} .
$$

Suppose that $\mathrm{g}(z)$ and $\mathrm{G}(z)$ are analytic functions in $\mathrm{E}$. Then $\mathrm{g}(z)$ is said to be subordinate to $\mathrm{G}(z)$ written as $\mathrm{g}(z) \prec \mathrm{G}(z)$ if and only if there exists $w(z)$, which is analytic in $\mathrm{E}$ with $w(0)=0$ and $|w(z)|<1$. Therefore, $g(z)=\mathrm{G}(w(z))$ belongs to $\mathrm{E} . \mathrm{g}(z)$ is univalent in $\mathrm{E}$ follows that $\mathrm{g} \prec \mathrm{G}$ is equivalent to $\mathrm{g}(0)=\mathrm{G}(0)$ and $\mathrm{g}(E) \subset \mathrm{G}(\mathrm{E})$.

The function $F(A, B, z)=\left(\frac{1+A z}{1+B z}\right)$ is the conformal mapping of the unit disk to circle, which is symmetric with respect to the real axis having center $\frac{1-A B}{1-B^{2}}$ and radius $\frac{A-B}{1-B^{2}}$ for each $A$, $B$ such that $-1 \leq B<A \leq 1$. A function $p(z)$ (with $p(0)=1$ ) is analytic in $E$ and belongs to the class $P[A, B]$ if $p(z)$ is subordinate to $F(A, B, z)$. In [5], Janowski introduced and investigated the class $P[A, B]$. Later on, Noor and Arif [8] investigated the class $P_{m}[A, B]$. They investigated that a function $p(z)$ (with $p(0)=1$ ) is analytic in $E$ and belongs to the class

(c) The Author(s) 2020. This article is licensed under a Creative Commons Attribution 4.0 International License, which permits use, sharing, adaptation, distribution and reproduction in any medium or format, as long as you give appropriate credit to the original author(s) and the source, provide a link to the Creative Commons licence, and indicate if changes were made. The images or other third party material in this article are included in the article's Creative Commons licence, unless indicated otherwise in a credit line to the material. If material is not included in the article's Creative Commons licence and your intended use is not permitted by statutory regulation or exceeds the permitted use, you will need to obtain permission directly from the copyright holder. To view a copy of this licence, visit http://creativecommons.org/licenses/by/4.0/. 
$P_{m}[A, B]$ if and only if there exist $p_{1}(z), p_{2}(z) \in \mathrm{P}[A, B]$ such that

$$
p(z)=\left(\frac{m}{4}+\frac{1}{2}\right) p_{1}(z)-\left(\frac{m}{4}-\frac{1}{2}\right) p_{2}(z)
$$

for $m \geq 2,-1 \leq B<A \leq 1$, and $z \in E$.

In [14], Rajapat et al. used the known family of fractional integral operators (with the Gauss hypergeometric function in the kernel) and defined new subclasses of strongly starlike and strongly convex functions of order $\beta$ and type $\alpha$ in the open unit disk $U$. Moreover, they established several inclusion relationships and interesting results associated with the fractional integral operators.

Shiraishi et al. [16] investigated some new sufficient conditions for the class of strong Caratheodory functions in the open unit disk $U$. Example and several corollaries of the main results were presented.

We consider a function $F_{\beta}(A, B, z)=\left(\frac{1+A z}{1+B z}\right)^{\beta}$ for $-1 \leq B \leq 1,-1 \leq A \leq 1(A \neq B)$, and $0<\beta \leq 1$, which is analytic and univalent in $E$. If $p(z)$ (with $p(0)=1$ ) is subordinate to $F_{\beta}(A, B, z)$, then the function $p(z)$ is analytic in $E$ and belongs to the class $\tilde{\mathrm{P}}^{\beta}[A, B]$.

Lemma $1.1([10])$ Suppose $f \in \mathcal{V}_{m}(\rho)$ for $m \geq 2$ and $0 \leq \rho<1$. Then $f(z)=\left(f_{1}(z)\right)^{(1-\rho)}$ for $f_{1}(z) \in \mathcal{V}_{m}$.

Lemma $1.2([6])$ Suppose $\xi=\xi_{1}+i \xi_{2}, \zeta=\zeta_{1}+i \zeta_{2}$ and $\Phi: D \subset C^{2} \rightarrow C$ is a complex-valued function, which satisfies the following conditions:

(i) $\Psi(\xi, \zeta)$ is continuous in a domain $D \subset C^{2}$,

(ii) $(1,0) \in \mathrm{D}$ and $\Phi(1,0)>0$,

(iii) $\operatorname{Re} \Phi\left(i \xi_{2}, \zeta_{1}\right) \leq 0$, whenever $\left(i \xi_{2}, \zeta_{1}\right) \in \mathrm{D}$ and $\zeta_{1} \leq-\frac{1}{2}\left(1+\xi_{2}^{2}\right)$.

If $q(z)=1+\sum_{n=1}^{\infty} c_{n} z^{n}$ is an analytic function in $E$ with $\left(q(z), z q^{\prime}(z)\right) \in D$ and $\operatorname{Re} \Phi(q(z)$, $\left.z q^{\prime}(z)\right)$ for $z \in E$, then $\operatorname{Re}(q(z))>0$, for $z \in E$.

Lemma $1.3([3])$ Suppose $r_{a} \leq \operatorname{Re}(a) \sin \left(\frac{\alpha \pi}{2}\right)-\operatorname{Re}(a) \cos \left(\frac{\alpha \pi}{2}\right), \operatorname{Im}(a) \geq 0$. Then the disk $\mid w-$ $a \mid \leq \mathrm{r}_{a}$ is contained in the sector $|\arg (w)| \leq \frac{\alpha \pi}{2}$ for $0 \leq \alpha<1$.

\section{Main results}

In this section, we discuss the coefficient problem, analytic property, inclusion results, and radius problem. It is interesting to mention that our obtained results are sharp as compared to the existing results in the literature.

First we define the following.

Definition 2.1 A function $q(z)$ (with $q(0)=1$ ) is analytic in $E$ and belongs to the class $\tilde{P}_{m}^{\beta}[A, B]$ (for $m \geq 2$ ) if and only if there exist $p_{1}(z), p_{2}(z) \in \tilde{\mathrm{P}}^{\beta}[A, B]$ such that

$$
q(z)=\left(\frac{m}{4}+\frac{1}{2}\right) q_{1}(z)-\left(\frac{m}{4}-\frac{1}{2}\right) q_{2}(z) \quad \text { for } z \in E
$$

It is easy to see that the set $\tilde{\mathrm{P}}_{m}^{\beta}[A, B]$ is convex. For special values of $A, B$, and $\beta$, we obtain several subclasses of analytic functions studied and investigated by several researchers [4, 7-9, 13-15]. 
Definition 2.2 An analytic function $f(z) \in \tilde{\mathrm{R}}_{m}^{\beta}[A, B]$ if and only if

$$
\frac{z f^{\prime}(z)}{f(z)} \in \tilde{\mathrm{P}}_{m}^{\beta}[A, B] \quad \text { for } z \in E
$$

Note that, for $\beta=1$, the class $\tilde{\mathrm{R}}_{m}^{\beta}[A, B]$ yields the class $\mathrm{R}_{m}[A, B][8]$ and $\tilde{\mathrm{R}}_{m}^{\beta}[1,-1]=\mathrm{R}_{m}(\beta)$. For $\beta=\frac{1}{2}, B=0$, and $A=a$ with $a \in(0,1]$, we obtain the class

$$
\tilde{\mathrm{R}}_{m}^{\frac{1}{2}}[a, 0]=q_{1}, q_{2} \prec \sqrt{1+a z} \text { for } z \in E .
$$

Definition 2.3 A function $f \in \mathcal{A}$ belongs to $\tilde{\mathrm{V}}_{m}^{\beta}[A, B]$ if and only if $f$ is locally univalent and

$$
\frac{\left(z f^{\prime}(z)\right)^{\prime}}{f^{\prime}(z)} \in \tilde{\mathrm{P}}_{m}^{\beta}[A, B] \quad \text { for } z \in E .
$$

From (2.1) and (2.3), it is considered that

$$
f \in \tilde{\mathrm{V}}_{m}^{\beta}[A, B] \quad \Leftrightarrow \quad z f^{\prime}(z) \in \tilde{\mathrm{R}}_{m}^{\beta}[A, B]
$$

Note that, for particular cases of $A, B$, and $\beta$, several classes are obtained and investigated in [8]. For $\beta=\frac{1}{2}, m=2, B=0$ and $A=a, a \in(0,1]$, Aouf [1] obtained the following class:

$$
\tilde{\mathbf{V}}_{m}^{\frac{1}{2}}[a, 0]=\left\{f \in A: \frac{\left(z f^{\prime}(z)\right)^{\prime}}{f^{\prime}(z)}=\left(\frac{m}{4}+\frac{1}{2}\right) q_{1}(z)-\left(\frac{m}{4}-\frac{1}{2}\right) q_{2}(z), q_{1}, q_{2} \prec \sqrt{1+a z}\right\} .
$$

Now we prove the following lemmas, which represent the coefficient inequality of functions belonging to the class $\tilde{\mathrm{P}}_{m}^{\beta}[A, B]$. By choosing special values of $A, B$, and $\beta$, we may relate our finding with the existing results. We shall assume $m \geq 2,-1 \leq B<A \leq 1$, $0<\beta \leq 1$, unless otherwise stated.

Lemma 2.4 Suppose $q(z)=1+\sum_{n=1}^{\infty} a_{n} z^{n}$ belongs to $\tilde{\mathrm{P}}_{m}^{\beta}[A, B]$. Then

$$
\left|a_{n}\right| \leq \frac{\beta m}{2}|A-B| \text { for all } n \geq 1
$$

Proof Suppose $q(z)$ belongs to $\tilde{\mathrm{P}}_{m}^{\beta}[A, B]$ with $q(z)=1+\sum_{n=1}^{\infty} a_{n} z^{n}$. Then

$$
\begin{aligned}
& q(z)=\left(\frac{m}{4}+\frac{1}{2}\right) q_{1}(z)-\left(\frac{m}{4}-\frac{1}{2}\right) q_{2}(z), \quad q_{1}, q_{2} \in \tilde{\mathrm{P}}^{\beta}[A, B] . \\
& \text { If } q_{1}(z)=1+\sum_{n=1}^{\infty} b_{n} z^{n} \text { and } q_{2}(z)=1+\sum_{n=1}^{\infty} c_{n} z^{n} \text {, then } \\
& 1+\sum_{n=1}^{\infty} a_{n} z^{n}=\left(\frac{m}{4}+\frac{1}{2}\right)\left(1+\sum_{n=1}^{\infty} b_{n} z^{n}\right)-\left(\frac{m}{4}-\frac{1}{2}\right)\left(1+\sum_{n=1}^{\infty} c_{n} z^{n}\right) .
\end{aligned}
$$

By comparing the coefficients of $z^{n}$ and using the triangle inequality, we get

$$
\left|a_{n}\right| \leq\left(\frac{m}{4}+\frac{1}{2}\right)\left|b_{n}\right|+\left(\frac{m}{4}-\frac{1}{2}\right)\left|c_{n}\right|
$$


Since $q_{i}(z) \prec\left(\frac{1+A z}{1+B z}\right)^{\beta}=1+\beta(A-B) z+\cdots$ for $i=1$, 2. Using the well-known result of Rogosinski [15] on subordination, we have $\left|b_{n}\right| \leq \beta|A-B|$ and $\left|c_{n}\right| \leq \beta|A-B|$ for all $n \geq 1$. This implies that

$$
\left|a_{n}\right| \leq\left(\frac{m}{4}+\frac{1}{2}\right) \beta|A-B|+\left(\frac{m}{4}-\frac{1}{2}\right) \beta|A-B|
$$

which is as required. This completes the proof.

The following lemma extends the inequality bound of functions in the class $\mathrm{P}_{m}[A, B]$.

Lemma 2.5 If $q(z)$ belongs to $\tilde{\mathrm{P}}_{m}^{\beta}[A, B]$ and $z=\mathrm{r} e^{i \theta}$, then

$$
\frac{m\left(k_{1}-k_{2}\right)+2\left(k_{1}+k_{2}\right)}{4\left(1-B^{2} r^{2}\right)} \leq \operatorname{Re}\{q(z)\} \leq \frac{m}{2}\left(\frac{1+A r}{1+B r}\right)^{\beta}
$$

where $k_{1}=\left(1-(A-B) r-A B r^{2}\right)^{\beta}, k_{2}=\left(1+(A-B) r-A B r^{2}\right)^{\beta}$.

Proof Suppose $q(z) \in \tilde{\mathrm{P}}_{m}^{\beta}[A, B]$. Then

$$
q(z)=\left(\frac{m}{4}+\frac{1}{2}\right) q_{1}(z)-\left(\frac{m}{4}-\frac{1}{2}\right) q_{2}(z), \quad \text { where } q_{1}(z), q_{2}(z) \text { in } \tilde{\mathrm{P}}^{\beta}[A, B]
$$

Using the triangle inequality, we have

$$
\operatorname{Re}(q(z)) \leq|q(z)| \leq\left(\frac{m}{4}+\frac{1}{2}\right)\left|q_{1}(z)\right|+\left(\frac{m}{4}-\frac{1}{2}\right)\left|q_{2}(z)\right|
$$

Since $q_{1}(z), q_{2}(z) \in \tilde{\mathrm{P}}^{\beta}[A, B]$, then

$$
\operatorname{Re}\left\{q_{i}(z)\right\} \leq\left(\frac{1+A|w|}{1+B|w|}\right)^{\beta}<\left(\frac{1+A r}{1+B r}\right)^{\beta} \text { for } i=1,2 \text {, }
$$

where $w(z)$ is defined in $E$, which is an analytic function with $w(0)=0$ and $|w(z)|<r$. Using (2.8) in (2.7), we get the right-hand side of (2.5).

Moreover, (2.6) can be written as

$$
\operatorname{Re}(q(z)) \geq\left(\frac{m}{4}+\frac{1}{2}\right) \operatorname{Re}\left\{q_{1}(z)\right\}-\left(\frac{m}{4}-\frac{1}{2}\right)\left|q_{2}(z)\right|
$$

Since $q_{1}(z), q_{2}(z) \in \tilde{\mathrm{P}}^{\beta}[A, B]$ and

$$
\operatorname{Re}\left\{q_{i}(z)\right\} \geq\left(\frac{1-A|w|}{1-B|w|}\right)^{\beta}>\left(\frac{1-A r}{1-B r}\right)^{\beta} \quad \text { for } i=1,2 \text {. }
$$

Using (2.10) in (2.9), we have

$$
\{\operatorname{Re} q(z)\} \geq\left(\frac{m}{4}+\frac{1}{2}\right)\left(\frac{1-A r}{1-B r}\right)^{\beta}-\left(\frac{m}{4}-\frac{1}{2}\right)\left(\frac{1+A r}{1+B r}\right)^{\beta}
$$


This implies that

$$
\begin{aligned}
\{\operatorname{Re} q(z)\} \geq & \left(\frac{m}{4}+\frac{1}{2}\right)\left(\frac{1-(A-B) r-A B r^{2}}{1-B^{2} r^{2}}\right)^{\beta} \\
& -\left(\frac{m}{4}-\frac{1}{2}\right)\left(\frac{1+(A-B) r-A B r^{2}}{1-B^{2} r^{2}}\right)^{\beta} .
\end{aligned}
$$

By simple calculations, we get the left-hand side of (2.5). Hence the proof.

Lemma 2.6 If a function $q(z) \in \mathcal{A}$ belongs to $\tilde{\mathrm{P}}_{m}^{\beta}[A, B]$, then $q(z) \in \mathrm{P}_{m}(\gamma)$, where $\gamma=\left(\frac{1-A}{1-B}\right)^{\beta}$ for $z \in E$.

Proof Let $q(z)$ belong to $\tilde{\mathrm{P}}_{m}^{\beta}[A, B]$. From (2.6) and (2.8), we have

$$
\operatorname{Re}\left\{q_{i}(z)\right\} \geq\left(\frac{1-A|w|}{1-B|w|}\right)^{\beta}>\left(\frac{1-A r}{1-B r}\right)^{\beta}, \quad i=1,2, z \in E
$$

From this it follows that each $q_{i}(z) \in \mathrm{P}(\gamma)$ for $i=1,2$, where $\gamma=\left(\frac{1-A}{1-B}\right)^{\beta}$ and $q(z) \in \mathrm{P}_{m}(\gamma)$ for $\gamma=\left(\frac{1-A}{1-B}\right)^{\beta}$. This implies that $\tilde{\mathrm{P}}_{m}^{\beta}[A, B] \subseteq \mathrm{P}_{m}(\gamma)$. Hence the proof.

In the following theorems, we discuss and investigate the coefficient problem, analytic property, inclusion results, and radius problem.

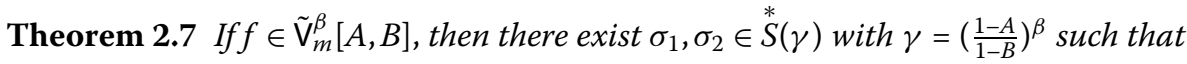

$$
f^{\prime}(z)=\frac{\left(\frac{\sigma_{1}(z)}{z}\right)^{\left(\frac{m}{4}+\frac{1}{2}\right)}}{\left(\frac{\sigma_{2}(z)}{z}\right)^{\left(\frac{m}{4}-\frac{1}{2}\right)}}, \quad z \in E .
$$

Proof Let $f(z)$ belong to $\tilde{\mathrm{V}}_{m}^{\beta}[A, B]$. Then $q(z)=\frac{\left(z f^{\prime}(z)\right)^{\prime}}{f^{\prime}(z)} \in \tilde{\mathrm{P}}_{m}^{\beta}[A, B]$. By Lemma 2.6, $\tilde{\mathrm{P}}_{m}^{\beta}[A, B] \subseteq$ $P_{m}(\gamma)$. This implies that $\frac{\left(z f^{\prime}(z)\right)^{\prime}}{f^{\prime}(z)} \in P_{m}(\gamma)$ with $\gamma=\left(\frac{1-A}{1-B}\right)^{\beta}$. It follows that $f \in V_{m}(\gamma)$. By Lemma 1.1, it has the form

$$
f^{\prime}(z)=(g(z))^{1-\lambda}, \quad \text { where } \lambda=\left(\frac{1-A}{1-B}\right)^{\beta} \text { and } g(z) \in \mathrm{V}_{m}
$$

Brannan [2] showed that each $g(z) \in \mathrm{V}_{m}$ has the representation of the form

$$
g^{\prime}(z)=\frac{\left(\frac{\mathrm{s}_{1}(z)}{z}\right)^{\left(\frac{m}{4}+\frac{1}{2}\right)}}{\left(\frac{\mathrm{s}_{2}(z)}{z}\right)^{\left(\frac{m}{4}-\frac{1}{2}\right)}}, \quad \mathrm{s}_{1}, \mathrm{~s}_{2} \in \stackrel{*}{ }
$$

It has been proved in [12] that each $\sigma_{i} \in \stackrel{*}{S}(\gamma)$ is

$$
\sigma_{i}(z)=z\left(\frac{\mathrm{s}_{i}(z)}{z}\right)^{1-\gamma}, \quad \mathrm{s}_{i}(z) \in \stackrel{*}{S}, i=1,2
$$

Using (2.14), (2.15), and (2.16), we get our required result (2.13). This completes the proof. 
Theorem 2.8 Let $f(z)$ belong to $\tilde{\mathrm{R}}_{m}^{\beta}[A, B]$ with $f(z)=z+\sum_{n=2}^{\infty} a_{n} z^{n}$ for $z \in E$. Then

$$
\left|a_{n}\right| \leq \frac{\left(\beta \frac{m}{2}|A-B|\right)_{n-1}}{(n-1) !} \text { for all } n \geq 2
$$

Proof Let $f(z)$ belong to $\tilde{\mathrm{R}}_{m}^{\beta}[A, B]$. Then $q(z)=\frac{z f^{\prime}(z)}{f(z)} \in \tilde{\mathrm{P}}_{m}^{\beta}[A, B]$. Suppose $q(z)=\frac{z f^{\prime}(z)}{f(z)}=1+$ $\sum_{n=1}^{\infty} c_{n} z^{n}$, where $q(z)$ is defined in $E$ and is analytic with $q(0)=1$. This implies

$$
z f^{\prime}(z)=f(z) q(z)
$$

The series representation of $(2.18)$ is

$$
z+\sum_{n=2}^{\infty} n a_{n} z^{n}=\left(z+\sum_{n=2}^{\infty} a_{n} z^{n}\right)\left(1+\sum_{n=1}^{\infty} c_{n} z^{n}\right)=z+\sum_{n=2}^{\infty} \sum_{i=0}^{n-1} c_{i} a_{n-i} z^{n}
$$

Comparing the coefficient of $z^{n}$, we obtain $(n-1) a_{n}=\sum_{i=1}^{n-1} c_{i} a_{n-i}, c_{0}=1$ and hence

$$
\left|a_{n}\right|=\frac{1}{n-1} \sum_{i=1}^{n-1}\left|c_{i}\right|\left|a_{n-i}\right| \quad \text { for } n \geq 2
$$

Since $q(z) \in \tilde{\mathrm{P}}_{m}^{\beta}[A, B]$ and using Lemma 2.4 , we get $\left|c_{i}\right| \leq \frac{\beta m}{2}(A-B)$ for all $\mathrm{n} \geq 1$. From (2.19), we have $\left|a_{n}\right| \leq \frac{\beta m(A-B)}{2(n-1)} \sum_{i=1}^{n-1}\left|a_{i}\right|$. In particular,

$$
\begin{aligned}
& \left|a_{2}\right| \leq \frac{\beta m}{2}(A-B), \\
& \left|a_{3}\right| \leq \frac{\beta \frac{m}{2}(A-B)}{2}\left(\frac{\beta m}{2}(A-B)+1\right)=\frac{\left(\frac{m}{2}(A-B)\right)_{2}}{(2) !} .
\end{aligned}
$$

For $n=\mathrm{j}$,

$$
\begin{aligned}
\left|a_{\mathrm{j}}\right| & \leq \frac{\beta \frac{m}{2}(A-B)}{\mathrm{j}-1} \sum_{i=1}^{j-1}\left|a_{i}\right|=\frac{\beta \frac{m}{2}(A-B)}{\mathrm{j}-1} \prod_{i=1}^{\mathrm{j}-2}\left(\frac{\beta \frac{m}{2}(A-B)}{i}+1\right) \\
& =\frac{\left(\beta \frac{m}{2}(A-B)\right)_{\mathrm{j}-1}}{(\mathrm{j}-1) !} \text { for } \mathrm{j} \geq 3,
\end{aligned}
$$

where we use the Pochhammer notation $(\beta)_{\mathrm{m}}$ introduced in [11]:

$$
(\beta)_{m}= \begin{cases}1, & m=0, \beta \in \mathbb{C} \backslash\{0\} \\ \beta(\beta+1) \cdot(\beta+m-1), & m \in \mathbb{N}, \beta \in \mathbb{C}\end{cases}
$$

Consider

$$
\left|a_{m+1}\right| \leq \frac{\beta \frac{m}{2}(A-B)}{m} \sum_{i=1}^{m-1}\left|a_{i}\right|+\frac{\beta \frac{m}{2}(A-B)}{m}\left|a_{m}\right|
$$


Using (2.20), we obtain the following:

$$
\begin{aligned}
\left|a_{\mathrm{j}+1}\right| & \leq \frac{\beta \frac{m}{2}(A-B)}{\mathrm{j}} \prod_{i=1}^{\mathrm{j}-2}\left(\frac{\beta \frac{m}{2}(A-B)}{i}+1\right)+\frac{\frac{m}{2}(\beta(A-B))^{2}}{\mathrm{j}(\mathrm{j}-1)} \prod_{i=1}^{\mathrm{j}-2}\left(\frac{\beta(A-B)}{i}+1\right) \\
& =\frac{\beta \frac{m}{2}(A-B)}{\mathrm{j}} \prod_{i=1}^{\mathrm{j}-1}\left(\frac{\beta \frac{m}{2}(A-B)}{i}+1\right)=\frac{\left(\beta \frac{m}{2}(A-B)\right)_{m}}{(\mathrm{j}) !} .
\end{aligned}
$$

By induction, we get (2.17). Hence the proof.

Theorem 2.9 Let $f(z)$ belong to $\tilde{\mathrm{V}}_{m}^{\beta}[A, B]$ with $f(z)=z+\sum_{n=2}^{\infty} a_{n} z^{n}, z \in E$. Then

$$
\left|a_{n}\right| \leq \frac{\left(\beta \frac{m}{2}(A-B)\right)_{n-1}}{(n) !} \quad \text { for } n \geq 2
$$

Proof Let $f(z)$ belong to $\tilde{\mathrm{V}}_{m}^{\beta}[A, B]$ with $f(z)=z+\sum_{n=2}^{\infty} a_{n} z^{n}$. Then $z f^{\prime}(z) \in \tilde{\mathrm{R}}_{m}^{\beta}[A, B]$. By using Theorem 2.8, we get

$$
\left|a_{n}\right| \leq \frac{\left(\beta \frac{m}{2}(A-B)\right)_{n-1}}{(n) !} \quad \text { for } n \geq 2
$$

Corollary 2.10 For $A=1, B=-1, f \in \tilde{\mathrm{V}}_{m}^{\beta}[1,-1] \equiv \tilde{\mathrm{V}}_{m}(\beta)$ and using Theorem 2.9 , we obtain

$$
\left|a_{n}\right| \leq \frac{(2 m \beta)_{n-1}}{(n) !} \quad \text { for } n \geq 2
$$

Theorem 2.11 If $f \in \tilde{\mathrm{V}}_{m}^{\beta}[A, B]$, then $f \in \tilde{\mathrm{R}}_{m}^{\beta}(\rho)$, where

$$
\rho=\frac{(2 \gamma-1)+\sqrt{(1-2 \gamma)^{2}+8}}{4}, \quad \gamma=\left(\frac{1-A}{1-B}\right)^{\beta} \text {. }
$$

Proof Suppose that

$$
\begin{aligned}
\frac{z f^{\prime}(z)}{f(z)} & =(1-\rho) q(z)+\rho \\
& =(1-\rho)\left\{\left(\frac{m}{4}+\frac{1}{2}\right) q_{1}(z)-\left(\frac{m}{4}-\frac{1}{2}\right) q_{2}(z)\right\}+\rho,
\end{aligned}
$$

where $q(z)$ is analytic in $E$ with $q(0)=1$. From (2.21), we have

$$
\frac{\left(z f^{\prime}(z)\right)^{\prime}}{f^{\prime}(z)}=(1-\rho)\left[q(z)+\frac{z q^{\prime}(z)}{(1-\rho) q(z)+\rho .}\right]+\rho .
$$

$f \in \tilde{\mathrm{V}}_{m}^{\beta}[A, B]$ implies that $\frac{\left(z f^{\prime}(z)\right)^{\prime}}{f^{\prime}(z)} \in \tilde{\mathrm{P}}_{m}^{\beta}[A, B]$ and $\tilde{\mathrm{P}}_{m}^{\beta}[A, B] \subseteq \mathrm{P}_{m}(\gamma)$, where $\gamma=\left(\frac{1-A}{1-B}\right)^{\beta}$. From this it follows that

$$
\frac{1}{1-\gamma}\left(\frac{\left(z f^{\prime}(z)\right)^{\prime}}{f^{\prime}(z)}-\gamma\right) \in P_{m}
$$


Using (2.23) and (2.24), we get

$$
\frac{(1-\rho)}{1-\gamma}\left[q(z)+\frac{\frac{1}{1-\rho} z \mathbf{q}^{\prime}(z)}{q(z)+\frac{\rho}{1-\rho} .}\right]+\frac{(\rho-\gamma)}{1-\gamma} \in P_{m}
$$

Now we define

$$
\Phi_{\eta_{1}, \eta_{2}}(z)=\frac{1}{\eta_{2}+1} \frac{z}{(1-z)^{\eta_{1}}}+\frac{\eta_{2}}{\eta_{2}+1} \frac{z}{(1-z)^{\eta_{1}+1}}
$$

with $\eta_{1}=\frac{1}{1-\rho}, \eta_{2}=\frac{\rho}{1-\rho}$. Using (2.22) with the convolution techniques given by Noor [7], we have

$$
\begin{aligned}
q(z)+\frac{\eta_{1} z \mathrm{q}(z)}{q(z)+\eta_{2}}= & (1-\rho)\left[\left(\frac{m}{4}+\frac{1}{2}\right)\left\{q_{1}(z)+\frac{\eta_{1} z \mathrm{q}_{1}^{\prime}(z)}{q_{1}(z)+\eta_{2} \cdot}\right\}\right. \\
& \left.-\left(\frac{m}{4}-\frac{1}{2}\right)\left\{q_{2}(z)+\frac{\eta_{1} z \mathrm{q}_{2}^{\prime}(z)}{q_{2}(z)+\eta_{2} \cdot}\right\}\right]+\rho .
\end{aligned}
$$

Thus, using (2.25) and (2.26), we have

$$
\frac{(1-\rho)}{1-\gamma}\left\{q_{i}(z)+\frac{\eta_{1} z \mathrm{q}_{i}^{\prime}(z)}{q_{i}(z)+\eta_{2} .}\right\}+\frac{(\rho-\gamma)}{1-\gamma} \in \mathrm{P} \quad \text { for } i=1,2 \text { and } z \in E \text {. }
$$

Since the first two conditions of Lemma 1.2 are obviously satisfied, we satisfy condition (iii) as follows:

$$
\begin{aligned}
\operatorname{Re} \Phi\left(i \xi_{2}, \zeta_{1}\right)= & \frac{\rho-\gamma}{1-\gamma}+\frac{1-\rho}{1-\gamma} \operatorname{Re}\left\{i \xi_{2}+\frac{\rho \zeta_{1}}{(1-\rho) i \xi_{2}+\rho}\right\} \\
& \leq \frac{\rho-\gamma}{1-\gamma}-\frac{1-\rho}{2(1-\gamma)}\left\{\frac{\rho\left(1+\zeta_{2}^{2}\right.}{(1-\rho)^{2} \xi_{2}^{2}+\rho^{2}}\right\}, \quad \text { for } \xi_{1} \leq-\frac{1+\xi_{2}^{2}}{2} \\
& =\frac{A+B \xi_{2}^{2}}{D},
\end{aligned}
$$

where

$$
\begin{aligned}
& A=2(\rho-\gamma) \rho^{2}-\rho(1-\rho), \\
& B=2(\rho-\gamma)(1-\rho)^{2}-\rho(1-\rho), \\
& D=2(1-\gamma)\left((1-\rho)^{2} u_{2}^{2}+\rho^{2}\right) .
\end{aligned}
$$

It is observed that (2.28) gives negative value for $A \leq 0$ and $B \leq 0$. Therefore, using $A \leq 0$, we have

$$
\rho=\frac{(2 \gamma-1)+\sqrt{(1-2 \gamma)^{2}+8}}{4}, \quad \gamma=\left(\frac{1-A}{1-B}\right)^{\beta},
$$

and from $B \leq 0$, we obtain $0 \leq \rho<1$. Hence all three conditions of Lemma 1.2 are satisfied. From this it follows that $q_{i}(z) \in \mathrm{P}$ for $i=1,2$ and $z \in E$. Therefore $q(z) \in \mathrm{P}_{m}$ and $f \in R_{m}(\rho)$, where $\rho$ is given in (2.29). This completes the proof. 
Corollary 2.12 If $\beta=\frac{1}{2}, B=0, A=a, a \in(0,1]$ and $f \in \tilde{\mathrm{V}}_{m}^{\frac{1}{2}}[a, 0]$. Then, using Theorem 2.11, we have $f \in \tilde{\mathrm{R}}_{m}^{\frac{1}{2}}(\rho)$, where

$$
\rho=\frac{(2 \gamma-1)+\sqrt{(1-2 \gamma)^{2}+8}}{4}, \quad \gamma=\sqrt{1-a}
$$

Theorem 2.13 If $f(z)$ belongs to $\tilde{\mathrm{R}}_{m}^{\beta}[A, B]$, then $f(z) \in \mathrm{R}_{m}(\gamma), \gamma=\left(\frac{1-A}{1-B}\right)^{\beta}$ for $z \in E$.

Proof $f(z)$ belongs to $\tilde{\mathrm{R}}_{m}^{\beta}[A, B]$ implies $q(z)=\frac{z f^{\prime}(z)}{f(z)}$ for some $q \in \tilde{\mathrm{P}}_{m}^{\beta}[A, B]$. Using Lemma 2.6, we get $\frac{z f^{\prime}(z)}{f(z)} \in \mathrm{P}_{m}(\gamma)$, where $\gamma=\left(\frac{1-A}{1-B}\right)^{\beta}$. Thus $f(z) \in \mathrm{R}_{m}(\gamma)$ for $z \in E$. This completes the proof.

Corollary 2.14 If $\beta=1, m=2$ and $f \in S^{*}[A, B]$. Then, using Theorem 2.13, we have $f(z) \in$ $S^{*}(\gamma)$, where $\gamma=\left(\frac{1-A}{1-B}\right)$.

\section{Conclusion}

We introduced some new classes of analytic functions with bounded radius and bounded boundary rotation by using the subordination. We discussed inclusion results, coefficient bounds, growth and distortion theorems of the classes. The radii of starlikeness and strong starlikeness of the classes have been computed. It is observed that most of the results are best possible.

\section{Acknowledgements}

Authors are thankful to the Editor-in-Chief Prof. Dr. Gradimir V. Milovanovic and all anonymous reviewers for their valuable comments towards the improvement of the paper. The second author is grateful to Air Marshal Javed Ahmed, HI(M) (Retd), Vice-Chancellor, Air University, Islamabad, Pakistan, for providing the excellent research facilities.

\section{Funding}

We have no funding for this project of research.

\section{Availability of data and materials}

Please contact the authors for data requests.

\section{Competing interests}

The authors declare that they have no competing interests.

Authors' contributions

All authors contributed equally to the manuscript, read and approved the final manuscript.

\section{Author details}

'Department of Mathematics, College of Science, Qassim University, Buraydah, Saudi Arabia. ${ }^{2}$ Department of Mathematics, Air University, Islamabad, Pakistan.

\section{Publisher's Note}

Springer Nature remains neutral with regard to jurisdictional claims in published maps and institutional affiliations.

Received: 14 April 2019 Accepted: 23 April 2020 Published online: 07 May 2020

\section{References}

1. Aouf, M.K., Dziok, J., Sokol, J.: On a subclass of strongly starlike functions. Appl. Math. Lett. 24, 27-32 (2011)

2. Brannan, D.A.: On functions of bounded boundary rotation I. Proc. Edinb. Math. Soc. (2) 16(4), 339-347 (1969)

3. Gangadharan, A., Ravichandran, V., Shanmugam, T.N.: Radii of convexity and strong starlikeness for some classes of analytic functions. J. Math. Anal. Appl. 211, 301-313 (1997)

4. Goodman, A.W.: Univalent Functions, Vols. I \& II. Polygonal Publishing House, Washington (1983)

5. Janowski, W.: Some extremal problems for certain families of analytic functions-I. Ann. Pol. Math. 28, $297-326$ (1973)

6. Miller, S.: Differential inequalities and Carathéodory functions. Bull. Am. Math. Soc. 81(1), 79-81 (1975)

7. Noor, K.I.: On radii of convexity and starlikeness of some classes of analytic functions. Int. J. Math. Math. Sci. 14(4), 741-746 (1991) 
8. Noor, K.I., Arif, M.: Mapping properties of an integral operator. Appl. Math. Lett. 25(11), 1826-1829 (2012)

9. Noor, K.I., Ul-Haq, W., Arif, M., Mustafa, S.: On bounded boundary and bounded radius rotations. J. Inequal. Appl. 2009 Article ID 813687 (2009)

10. Padmanabhan, K.S., Parvatham, R.: Properties of a class of functions with bounded boundary rotation. Ann. Pol. Math 31(3), 311-323 (1975)

11. Petojevic, A.: A note about the Pochhammer symbols. Math. Morav. 12(1), 37-42 (2008)

12. Pinchuk, B.: On starlike and convex functions of order $\alpha$. Duke Math. J. 35, 721-734 (1968)

13. Pinchuk, B.: Functions of bounded boundary rotation. Isr. J. Math. 10, 6-16 (1971)

14. Prajapat, J.K., Raina, R.K., Srivastava, H.M.: Some inclusion properties for certain subclasses of strongly starlike and strongly convex functions involving a family of fractional integral operators. Integral Transforms Spec. Funct. 18(9), 639-651 (2007)

15. Rogosinski, W.: On the coefficients of subordinate functions. Proc. Lond. Math. Soc. (2) 48, 48-82 (1943)

16. Shiraishi, H., Owa, S., Srivastava, H.M.: Sufficient conditions for strongly Caratheodory function. Comput. Math. Appl. 62, 2978-2987 (2011)

\section{Submit your manuscript to a SpringerOpen ${ }^{\circ}$ journal and benefit from:}

- Convenient online submission

- Rigorous peer review

- Open access: articles freely available online

- High visibility within the field

- Retaining the copyright to your article

Submit your next manuscript at $\gg$ springeropen.com 